\title{
Research Methodology on Language Development from a Complex Systems Perspective
}

\author{
DIANE LARSEN-FREEMAN \\ English Language Institute \\ 500 E. Washington St. \\ University of Michigan \\ Ann Arbor, MI 48104 \\ Email: dianelf@umich.edu
}

\author{
LYNNE CAMERON \\ The Open University \\ Centre for Language and Communication \\ Walton Hall \\ Milton Keynes \\ MK7 6AA \\ United Kingdom \\ Email: l.j.cameron@open.ac.uk
}

\begin{abstract}
Changes to research methodology motivated by the adoption of a complexity theory perspective on language development are considered. The dynamic, nonlinear, and open nature of complex systems, together with their tendency toward self-organization and interaction across levels and timescales, requires changes in traditional views of the functions and roles of theory, hypothesis, data, and analysis. Traditional views of causality are shifted to focus on co-adaptation and emergence. Context is not seen as a backdrop, but rather as a complex system itself, connected to other complex systems, and variability in system behavior takes on increased importance. A set of general methodological principles is offered, and an overview of specific methods is given, with particular attention to validity in simulation modeling.
\end{abstract}

IN THIS ARTICLE, WE WISH TO CONSIDER ways of researching language development from a complex systems perspective. ${ }^{1}$ A complex system is one that emerges from the interactions of its components. The components can be agents or elements. An example of the former would be a flock of birds, which emerges from the interactions of the individual birds that compose it. An example of the latter would be air currents, moisture, and temperature interacting to yield a weather system. Complex systems are often heterogeneous, being made up of both agents and elements. The ecosystem of a forest would include, as component agents, animals, birds, insects, and people; component elements would include trees, winds, rainfall, sunshine, air quality, soil, and rivers. Not only are there many agents and elements in a complex system like an ecosystem, but they are of different kinds.

Some complex systems, such as the stock market, are dynamic and nonlinear. They are dy-

The Modern Language Journal, 92, ii, (2008)

0026-7902/08/200-213 \$1.50/0

(C)2008 The Modern Language Journal namic, in that the worth of stocks changes over time, sometimes continuously, both up and down. They are nonlinear, in that at other times, the systems change suddenly and discontinuously. These systems are open to outside influences; in the case of the stock market, it is open to new investments or to changes in regulations. Following a precipitous decline in the stock market, if new investments are forthcoming, the system is said to selforganize, generating new, emergent modes of behavior from the investments that are being made. ${ }^{2}$

Chaos/complexity theory has been used to study complex, dynamic, nonlinear, open systems, including naturally occurring systems, such as the weather and the rise and fall of animal populations. More recently, it has also been applied to human behavior in, for example, the disciplines of economics and epidemiology. Although predator-prey interactions influencing animal populations and the spread of disease are, of course, different in many ways, they have in common dynamical properties of change.

Many of the phenomena of interest to applied linguists can be seen as complex systems as well (Larsen-Freeman, 1997; Cameron, 2003). 
Applied linguistic complex systems are likely to contain many subsystems, nested one within another. For example, if we see the speech community as a complex system, then it will also have within it sociocultural groups that themselves function as complex systems; individuals within these subgroups can be seen as complex systems, as can their individual brain systems. There are complex systems at all levels, from the social level to the neurological levels. The complexity of a complex system arises from components and subsystems being interdependent and interacting with each other in a variety of different ways.

Because applied linguists deal with complex dynamic systems, we think complexity theory offers a helpful way of thinking about applied linguistic matters (Larsen-Freeman, 1997; Larsen-Freeman \& Cameron, 2008; see also de Bot, Lowie, \& Verspoor, 2007; Ellis \& Larsen-Freeman, 2006). We use complexity theory as an umbrella term to include not only complexity theory, but closely related chaos theory, dynamic(al) systems theory, and complex systems theory. It also relates well and therefore embraces ecological approaches that adopt an analogy between complex ecological systems and human language using/learning systems (Kramsch, 2002; Leather \& van Dam, 2003; van Lier, 2004). Although complexity theory has originated in and benefited greatly from work in the natural sciences, there is certainly a material difference between applied linguistics and the natural sciences with respect to objects of inquiry. However, the question we entertain in this article is whether the difference is as clear when it comes to "methods of inquiry" (Gaddis, 2002, p. 113). ${ }^{3}$ To address this question, we will first discuss how complexity theory warrants changes from traditional research that tests hypotheses in order to identify causes for particular events. Then, we will offer some general methodological principles for investigating language development from a complexity theory perspective. Last, we will make some suggestions for how to take extant methods and apply the new principles to them so that they are relevant for a new ontology. Central to all aspects of these discussions is the dynamic nature of complex systems-change and variability become the heart of what is investigated.

\section{CHANGES FROM TRADITIONAL RESEARCH}

\section{The Nature of Hypotheses}

A first major area of difference between a complex systems and a traditional approach to research involves the nature of hypotheses and theories that drive or frame research. Specifically, it has to do with how we understand and attempt to explain the phenomena that are observed-the nature of explanations and the level of explanations. Complexity theory works at the system level, and explanation is in terms of the system's behavior, not at the level of individual agents or elements (unless, of course, they are the system that is under investigation). Because many complex systems are interconnected and coordinated, it is not always possible to explain behavior, and changes in behavior, by detailing their separate components and roles in what Clark (1997) called "a componential explanation" (p. 104). To use an analogy that may be familiar to readers, when a sand pile is being steadily added to from above, it eventually collapses in a large avalanche, but we can never know which particular grain of sand will produce the avalanche (Bak, 1997). What we know is that if sand keeps being added to the pile, eventually an avalanche will occur. We also know about patterns and distributions of avalanche sizes. Because we know these things, we can articulate an explanation at a higher level, that is, our explanation of sand pile avalanches is expressed in terms of the structure and stability of the sand pile, rather than in terms of the behavior of individual grains of sand.

Such a perspective is antithetical to the common reductionist approach in science, which relies on the central principle that one can best understand an object of inquiry by taking it apart and examining its pieces. From a complexity theory perspective, knowing about the parts individually is insufficient because complexity theorists are interested in understanding how the interaction of the parts gives rise to new patterns of behavior. In addition, reductionist explanations can never be exhaustive or complete because the behavior of every part of the system cannot be fully known. Moreover, even if it were possible to know about the behavior of the individual parts and their interactions, the individual parts do not make a consistent contribution to interactions over time. Furthermore, the systems can be very sensitive. This last point is often referred to as the butterfly effect, the notion that even a small action, such as a butterfly flapping its wings in one part of the world, can have a large influence on meteorological conditions somewhere else. The "unknowableness" in complex systems, together with their nonlinearity, which leads to discontinuity and self-organizing change, makes them unpredictable in the conventional sense of predictability.

Systems and behavior can, of course, be described retrospectively, after change has happened, and this is the central work of a complexity theory approach. What we can observe 
in language development is what has already changed-the trajectory of the system. This is a trace of the real system, from which we try to reconstruct the elements, interactions, and change processes of the system (Byrne, 2002). Such a process is retrodiction (or retrocasting), rather than prediction (or forecasting); that is, it explains the next state by the preceding one.

In conventional science, explanation produces prediction in the form of testable hypotheses. In complexity theory, after a system has changed or evolved, the process might be explicable through an appeal to such notions as self-organization, but new predictions are not necessarily a consequence. Of course, we may have expectations of how a process will unfold, or even of its outcomes, based on prior experience, but essentially, adopting a complexity theory perspective brings about a separation of explanation and prediction.

\section{Causality}

Closely related, because a key type of scientific explanation is that "cause $\mathrm{x}$ produces outcome $y$," is the matter of causality. In the traditional reductionist scenario, the researcher searches for a critical "element whose removal from a causal chain would alter the outcome" (Gaddis, 2002, p. 54) and that can thus be said to be the cause of the outcome. To suggest that an event may have had many antecedents or causes is not considered good research. Gaddis (2002) quoted a recent guide to social science research to illustrate how this traditional view of causality translates into methodology: "A successful project is one that explains a lot with a little. At best, the goal is to use a single, explanatory variable to explain numerous observations on dependent variables" (p. 55). He then explained that "reductionism implies, therefore, that there are indeed independent variables, and that we can know what they are" (p. 55)-a concept that entertaining language development as a complex system would encourage us, at least, to question because the unknowableness and interconnectedness of systems makes it much more difficult, if not impossible, to isolate independent variables that act in causative ways.

In fact, some would go even further. Byrne (2002) argued that social scientists' adoption of "variable centred analysis," in which some variables are held to work as causative or determining forces, is ill-advised. He urged: "death to the variable," and then continued:

Let us understand clearly, once and for all, that variables don't exist. They are not real. What exists are complex systems, which systems are nested, intersect- ing, which involve both the social and the natural, and which are subject to modification on the basis of human action, both individual and social. (p. 31)

Although dealing a death blow to variables may be more extreme than some would like, and indeed we have preserved here the use of the term variable (though we redefine it as a collective variablesee "Methodological Principles" section), there is no doubt that complexity theory types of explanation, which do not allow prediction in the traditional sense, differ from what we are used to. Instead of investigating single variables, we study modes of system change that include selforganization and emergence. Emergent properties or phenomena occur when change on one level of social grouping or on the timescale of a system leads to a new mode on another level or timescale. An applied linguistics example is when a new lexical item is first used in conversations between individuals. As this behavior iterates, and others adapt to it, the new lexical item later becomes established in language use more generally. Eventually, it may become sufficiently conventionalized to warrant a dictionary entry. Cameron and Deignan (2006) cited the example of emotional baggage, a metaphorical phrase referring to longterm issues that are still active in a person's mind. This phrase has emerged fairly recently in English, influenced by social changes and language uses. Its emergence could not have been predicted using the usual definition of prediction; nevertheless, the genealogy of such phrases can be studied and their origin can sometimes be explained in retrospect.

We have termed one type of causality that is operable in complexity theory co-adaptation (LarsenFreeman \& Cameron, in press). Co-adaptation describes a kind of mutual causality, in which change in one system leads to change in another system connected to it, and this mutual influencing continues over time. For example, a native speaker may adjust pronunciation, speed, and lexicogrammar when speaking with a nonnative speaker, and the nonnative speaker may adjust in response as the language she or he is hearing becomes easier to process. Co-adaptation, of course, occurs in first language development, as well, between an infant and an "other," who, early on, is his or her caretaker. As the child and the caretaker interact, the language resources of each are dynamically altered, as each adapts to the other. In classrooms, teachers and students continually co-adapt—establishing the patterns of familiar routines and activities. From co-adaptation of teacher and student behaviors emerges a 
structure at another level, one that we might call the lesson. Similarly, moving down one level, within the student as an individual, co-adaptation of multiple subsystems of language leads to the emergence of new language resources with which to communicate.

Van Geert and Steenbeek (in press) described a particular type of co-adaptation when they appealed to the notion of superposition, in which a phenomenon is characterized by two (apparently) incompatible properties at the same time. Their example is the construct of intelligence, which is, they argued, "at the same time (almost) completely determined by the environment and (almost) completely determined by genes" (p. 5). This apparent paradox is resolved when it is seen that "genes and environment are locked in a complex chain of steps over time and that they cannot be conceived of as variables that make mutually independent contributions to development" (p. 5). Van Geert and Steenbeek (in press; see also van Geert, this issue) used the idea of mutual causality in modeling first language development. They saw this development as

a web of interacting components that entertain supportive, competitive and conditional relationships. The relationships are reciprocal but not necessarily symmetrical. For instance, it is likely that an earlier linguistic strategy bears a supportive relationship to a later, more complex linguistic strategy. The latter, however, may have a competitive relationship with its predecessor.... By modeling such webs of reciprocal action, it is possible to understand the emergence of stages, temporary regressions, inverse U-shaped growth and so forth. (p. 9)

Following a suggestion from Gaddis (2002), it may be helpful to think of causation as contingent, rather than categorical. In other words, we should think in terms of "particular generalizations" (p. 62), not universal generalizations. We might acknowledge tendencies or patterns, but resist claiming applicability for our findings beyond specific times and places. For example, if asked whether a particular teaching technique is effective, a teacher's reply, "It all depends," is particularly apt to illustrate this point, because the success of a given technique depends on many things. It depends on the characteristics and goals of the particular individuals who compose the class. It depends on the school and community in which the class is situated. It depends on the day of the week that the technique is used, even on the time of the day, and so forth. If we think in terms of reality as a web, then "everything is connected in some way to everything else" (Gaddis, 2002, p. 64). The independence of any individual variable then becomes questionable, as does the idea of a single cause giving rise to a complex event. Rather, it is likely that there are multiple and interconnected causes underlying any shift or outcome. "We may rank their relative significance, but we'd think it irresponsible to seek to isolate-or 'tease out'single causes for complex events" (Gaddis, 2002, p. 65). Of course, because the future is unknowable, it does not follow that there is no continuity from the past. "Some continuities will be sufficiently robust that contingencies will not deflect them" (p. 56).

In summary, a complexity theory perspective, by deconstructing the very notion of causality, undoes the conventional expectation that a good theory is one that describes, explains, and predicts. Instead of static laws and rules that social scientists and applied linguists, in following research in the natural sciences, have traditionally sought to uncover, we are faced with tendencies, patterns, and contingencies. Instead of single causal variables, we have interconnecting and self-organizing systems that co-adapt and that may display sudden discontinuities and the emergence of new modes and behaviors. A good application of complexity theory describes the system, its constituents, their contingencies, and also their interactions. Teasing out the relationships and describing their dynamics are key tasks of the researcher working from a complex systems perspective.

\section{Data and Evidence}

A complexity theory view of language use and language development has implications for research methodology beyond the nature of hypotheses, causality, and predictions. What data do we collect and what kind of measurements are to be made, in a perspective where everything is connected and continually changing? Adopting a complexity theory perspective shifts our view of what seems to need empirical investigation, in particular the role of context and environment. It changes what we need to collect as data to understand a complex system, in particular our attitude to variation. It changes what we notice in the behavior of systems: Flux and variability signal possible processes of self-organization and emergence; sudden phase shifts signal important changes and can direct attention to the conditions that lead up to them. In this section of this article, we consider the implications for research of three key complexity aspects: stability and variability, context, and interacting levels and scales.

Stability and Variability. In complexity theory, phenomena of interest are visualized by evoking 
spatial metaphors. For instance, the state space of a complex system is a multidimensional landscape that represents all possible states of the system. As a complex system makes its way through state space, it is attracted to or prefers a particular region of that space. The trajectory of the system over its state space thus represents the actual successive states of the system. Even when a complex system is in a stable mode (called an attractor), it is still continually changing as a result of change in its constituent elements or agents and change in how they interact, and in response to change in other systems to which it is connected. In other words, stability is not stasis. A complex system will show degrees of variability around stabilities, and the interplay of stability and variability offers potentially useful information about change in the system. From this perspective, variability in data is not noise to be discarded when averaging across events or individuals, or the result of measurement error (van Geert \& van Dijk, 2002), but is part of the behavior of the system, to be expected around stabilities, and particularly at times of transition from one phase or mode of behavior to another. Changes in variability can be indicators of development. If we smooth away variability, by averaging, for example, we lose the very information that may shed light on emergence (Larsen-Freeman, 2006a). If, instead, we pay attention to the nature of changes in stability and variability, we may find new ways of understanding language learning or development processes. As Fischer and Bidell (1998) suggested when they discussed cognitive development:

The static notion of stage structure, which dominated theories of cognitive development from its inception as a field of study through the early 1980s, has proven incapable of accounting for the massive and growing evidence of both variation and consistency: wideranging variability within and across individuals in the age of acquisition of logical concepts across domains and contexts, systematic sequences in the order of acquisition of many of these concepts and their components, and high synchrony in development of some concepts under some conditions.... Why have there been so few efforts to account for systematic variability in developmental patterns? (p. 470)

We might ask the same question about patterns of language development.

Variability and its relations with stability can be measured in two ways. First, the degree of variability around the stable mean serves as "an index of the strength of the behavioral attractor" (Thelen \& Smith, 1994, pp. 86-87). If variability increases, with concurrent loss of stability, the system may be about to enter a transition to a new mode. An- other measure uses the outcome of perturbing, or pushing, the system away from its stable behavior. The more stable the system is, the more likely it is to return to the stable mode. A less stable system is more likely to shift into a different behavior. Around times of transition, the system will be more easily perturbed when pushed out of its path (Thelen \& Smith, 1994).

Lack of stability in knowledge or performance may suggest something interesting happening in the language or language development system. Investigation of variability occurs in dynamic modeling (see van Geert, this issue) and can indicate potentially fruitful points in longitudinal data for in-depth investigation.

The Changed Nature of Context. In a complexity perspective, context includes the physical, social, cognitive, and cultural, and is not separable from the system. Context cannot, for example, be seen as a frame surrounding the system that is needed to interpret its behavior (Goffman, 1974). The connection between system and context is shown by making contextual factors parameters or dimensions of the system. Complex systems are often sensitive to changes in context and adapt dynamically to them in a process of "soft assembly" (Thelen \& Smith, 1994, p. 60). For example, the galloping motion of the complex system formed by a horse and its rider will be soft assembled as the horse adapts to changes in the position of the rider, the firmness of the ground surface, wind speed and direction, and its own fitness and health. Similarly, the children observed learning to reach and grasp by Thelen (Thelen \& Smith, 1994) adapted their movement dynamically to the local conditions of each task, such as the slope of the surface or the distance at which an object was placed. Furthermore, these local adaptations to contextual conditions are the foundation for emergent change, that is, development, on a longer timescale. From repeated adaptive experiences in the "here-and-now" of context, attractors emerge in the system that represent a more global order, that is, at a higher level of social organization or longer timescale.

In our applied linguistics context, any use of language can be seen as the soft assembly of language resources in response to some languageusing activity. Use of language need not be verbal production but would include any activity, inside or outside the classroom, that involves mental activity around language: understanding, speaking, recall of language, meaningful practicing, and so on. Language learning or development emerges with these adaptive experiences of language use. 
The context of a language learning or languageusing activity includes the intrinsic dynamics of the learner, that is, what individuals bring to the activity, for example, their cognitive context (e.g., working memory); the cultural context (e.g., what roles the teacher and the students play in this culture); the social context, including relationships with other learners and the teacher; the physical environment; the pedagogical context, that is, the task or materials, and the sociopolitical environment; and so on. Many of these contextual conditions also will be complex, dynamic, adaptive systems. The students in action will soft assemble their language and other resources in response to these contextual conditions, and the teacher and other students (to the extent that they can) will adapt in response to the students' actions. We thus cannot separate the learner or the learning from context in order to measure or explain them. Rather we must collect data about and describe all the continually changing system(s) involved. In holding learner and context as inseparable, a complexity theory perspective makes a similar argument to sociocultural (e.g., Lantolf, 1994) and ecological approaches (e.g., van Lier, 2004), but perhaps emphasizes a different facet, seeing learner and a complex context as interacting, co-adaptive dynamic systems.

The complexity theory perspective also puts a slightly different slant on the claim that learning, as change, is at once individual and social. Each individual is unique because he or she has developed his or her physical, affective, and cognitive self from a different starting point and through differing experience and history. Each individual thus acts as a unique learning context, bringing a different set of systems to a learning event, responding differently to it, and therefore, learning differently as a result of participating in it. In averaging across individuals, we lose detailed information about how those systems change in response to changes in context. When an individual participates in a group, the group as a system both affects and is affected by the individual. So, to understand language learning processes, we need to collect data about individuals (as well as about groups), and about individuals as members of groups as well as working alone. When researching groups, we need to see them as interconnecting systems of individuals. As van Geert and Steenbeek (in press) said:

Although it is statistically possible to separate contextand person-aspects, such separation requires the assumption of independence of persons and contexts. This assumption is untenable under a dynamic interpretation of performance. On a short time scale, context affordances ${ }^{4}$ and person abilities result from the real-time interaction between the two and are, therefore, inherently dependent on one another. On a longer time scale, persons tend to actively select and manipulate the contexts in which they function, whereas contexts on their turn help shape the person's characteristics and abilities.

Nested Levels and Timescales. One other special consideration must be made in studying language and its development from a complex systems perspective, and that is the matter of nested levels and timescales. To start with nested levels, from a complexity theory, systems exist at different levels of granularity; that is, they are nested from a macrolevel, such as that of a whole ecosystem, all the way down to a microlevel, such as subatomic particles. Such scale hierarchies are well known in biology (Salthe, 1993). The various systems operate at different levels but are interconnected. When attempting to explain the behavior of the system, each of these levels may contribute. In this example, cited by Sealey and Carter (2004), the spread of tuberculosis is explained through invoking four nested, interacting levels:

In accounting for who gets $\mathrm{TB}$ and in what contexts, Byrne argues, we must first acknowledge a biological aetiology (people must be exposed to the bacillus) and a genetic component (some people will have a natural resistance to the bacillus). However, whether particular individuals contract the disease or not will be socially contingent, since it will depend on the interaction between these features and other levels of the social world. Byrne identifies four such levelsthe individual, the household, the community and the nation-state-existing in a nested hierarchy. (p. 198)

In addition to nested levels, the complex systems researched by applied linguists operate on a range of timescales, from the milliseconds of neural processing through the minutes of a classroom activity to change on an evolutionary timescale. For a particular study, certain levels and scales will be focal, but they will be affected by what happens on other levels and scales. As Lemke (2002) pointed out, "certain events widely separated in linear time may be more relevant to meaningful behavior now than other events which are closer in linear time" (p. 80). Because activity on one level and scale influences what happens on other levels and scales, with phenomena sometimes emerging at a particular level or scale as a result of activity at a lower level or in an earlier period, it is important when we are conducting research within a complex systems approach that we seek to find relationships within and across different levels and timescales. When 
we are able to do so, the results will be all the more powerful.

\section{Summary}

To summarize thus far: In complexity theory, we search for ways to access the relational nature of dynamic phenomena, a search that is not the same as the pursuit of an exhaustive taxonomy of factors that might account for behavior of any given phenomenon. Furthermore, we attempt to distinguish between contingent and necessary outcomes. Thus, the nature of description and explanation changes, cause and effect no longer operate in the usual way, and reductionism does not produce satisfying explanations that are respectful of the interconnectedness of the many nested levels and timescales that exist.

\section{METHODOLOGICAL PRINCIPLES FOR RESEARCHING LANGUAGE AND LANGUAGE DEVELOPMENT}

From this complexity theory perspective, certain methodological principles follow. In the complexity approach that we adopt, it is important

1. To be ecologically valid, including context as part of the system(s) under investigation;

2. To honor the complexity by avoiding reductionism, and to avoid premature idealization by including any and all factors that might influence a system;

3. To think in terms of dynamic processes and changing relationships among variables, by considering self-organization, feedback, and emergence as central;

4. To take a complexity view of reciprocal causality, rather than invoking simple, proximate cause-effect links;

5. To overcome dualistic thinking, such as acquisition versus use or performance versus competence, and to think in terms of co-adaptation, soft assembly, and so forth;

6. To rethink units of analysis, identifying collective variables (those variables that characterize the interaction among multiple elements in a system, or among multiple systems, over time);

7. To avoid conflating levels and timescales, yet seek linkages across levels and timescales, and include thinking heterochronically; and

8. To consider variability as central, and investigate both stability and variability in order to understand the developing system.

\section{MODIFIED RESEARCH METHODOLOGIES}

We now move to consider practical implications of the complexity theory perspective for the empirical investigation of language development, such as measuring the effectiveness of pedagogic interventions or tracking stabilities and variation in learner language. When enacted, some of the methodological principles cited previously will no doubt lead to innovations of which we are currently unaware. Some methods, however, are already in existence with designs that make them useful for investigating complex systems. Other methods will need some modification.

\section{Ethnography}

In many ways, qualitative research methods, such as ethnography, would appear to serve the understanding of language as a complex dynamic system well, in that they "attempt to honor the profound wholeness and situatedness of social scenes and individuals-in-the-world" (Atkinson, 2002, p. 539), by studying real people in their human contexts and interactions, rather than aggregating and averaging across individuals as happens in experimental and quantitative studies. Atkinson cited as examples of applicable ethnographic methods the works of Holliday (1996), Davis and Lazaraton (1995), and Ramanathan and Atkinson (1999).

Agar (2004) went further, arguing that ethnography is itself a complex adaptive system, that evolves and adapts as the researcher uses it:

[It] will lead you to ways of learning and documenting that you had no idea existed when you first started the study. You will learn how to ask the right question of the right people in the right way using knowledge you didn't know existed. You will see that certain kinds of data belong together in ways that you would never have imagined until you'd worked on the study for awhile... methods "evolve" as local information about how to do a study accumulates. Ethnography does this. Traditional research prohibits it. (p. 19)

It is important to note that ethnographers seek emergent patterns in what they study. Agar suggested that ethnography is a fractal-generating process. What ethnographers are looking for are processes that apply iteratively and recursively at different levels to create patterns, variations that emerge from adaptation to contingencies and environment.

One possible modification of ethnographic method, from a complexity theory perspective, however, is the assumption that, when applied 
effectively, ethnography can produce objectivity. From our perspective, no matter how a researcher tries, total objectivity - a view of matters apart from who he or she is-can never be achieved. A complex system is dependent on its initial conditions, and these conditions include the researcher. Accounts of the "same" phenomenon will differ when produced by different ethnographers (Agar, 2004). This finding, of course, is not a problem, just a fact.

\section{Formative and Design Experiments/Action Research}

Conventional experiments are problematic from a complexity theory perspective because of their ecological invalidity. Furthermore, they can only, at best, lead to claims about proximate, linear causes, while not allowing for multiple or reciprocally interacting variables, which change over time. Although we would not wish to discount experimental claims, we need to problematize them in light of the fact that any cause and effect link that is found might actually occlude fundamental nonlinearity (Larsen-Freeman, 1997). Who can say, for example, on the basis of a pretest/posttest design that a particular experimental treatment works or does not work? If the results are not significant, the effects of the treatment may not yet be manifest; if the results are significant, they may have followed from an experience prior to the pretest. A further limitation of conventional experiments occurs when researchers attempt to control context and situation, rather than investigating adaptation to the unique particularities of context: "They try to ensure that an intervention is implemented uniformly despite different circumstances; and they focus on post intervention outcomes instead of what happens while the intervention is implemented" (Reinking \& Watkins, 2000, p. 384).

Qualitative and ethnographic studies, which carefully document instructional practices, are the means traditionally looked to in order to offset these limitations. However, these studies often fail to tease out factors that affect success or failure in educational interventions, and then fail to explore how interventions might be adapted in response to those factors to be more effectively implemented (Reinking \& Watkins, 2000).

A different type of experiment, called a formative experiment (Jacob, 1992, as cited in Reinking $\&$ Watkins, 2000), focuses on the dynamics of implementation, using the ideas of soft assembly and co-adaptation, and might thus be capable of overcoming these limitations. Using Newman's (1990) words, Reinking and Watkins defined it as follows: "In a formative experiment, the researcher sets a pedagogical goal and finds out what it takes in terms of materials, organization, or changes in the intervention in order to reach the goal" (Newman, 1990, as cited in Reinking \& Watkins, 2000, p. 388). This (neo-) Vygotskyan idea appears to be compatible with a complex systems perspective. Formative experimenters attempt to investigate the potential of a system rather than its state; they accept that change in one system can produce change in other connected systems; they attempt to describe the interconnected web of factors influencing change; and they investigate processes of co-adaptation in response to changed pedagogic goals.

Another research method that has some characteristics that are compatible with complexity theory is what has been called design-based research or a design experiment. Barab (2006) explained that, in complex learning environments, it is difficult to test the causal impact of particular variables with experimental designs. Design-based research "deals with complexity by iteratively changing the learning environment over time-collecting evidence of the effect of these variations and feeding it recursively into future design" (p. 155). Lobato (2003) discussed how design experiments differ from traditional experiments in that the focus of investigation is shifted from the products or outcomes of learning to learning processes. Teachers are encouraged to respond flexibly to what is taking place in the classroom, as they might ordinarily do, not to follow some experimental treatment protocol. In this way, a design experiment shifts "from a reductionist cognitive view of learning to one that is also social in nature" (Lobato, 2003, p. 19).

Better known, perhaps, is action research. Although often motivated by sociopolitical reasons (Kemmis, 2001), action research is also concerned with possibility rather than prediction, and with the study of systems. Its researchers, who may be practitioners rather than outside experimenters, deliberately introduce "noise" into the system to see what transpires. They choose a problem in their teaching which they study and to which they apply the Lewinian cycle of diagnosing/action planning/action taking/evaluating/specifying learning (Baskerville \& Wood-Harper, 1996). Action research takes place in the system environment, and investigation of the system's response to a perturbation contributes to a deeper understanding of the system dynamics. 
Longitudinal, Case-Study, Time-Series Approaches

Another methodology that might be useful, when modified for complex system purposes, is a longitudinal, case-study, time-series approach, which enables connections to be made across levels and timescales. In contrast, often interlanguage studies tend to be cross-sectional, denying us the idiographic description of individual growth and variability.

It is not sufficient simply to lengthen the amount of time that behavior is sampled. There is a need to identify appropriate timescales on which data are collected-does the change show itself over a period of days, or months, or does it take a lifetime? (Ortega \& Iberri-Shea, 2005). There is also a need to select appropriate sampling intervals, which will depend on the rate of change. Willett (1994) suggested that a researcher

must assemble an observed growth record for each person in the dataset. If the attribute of interest is changing steadily and smoothly over a long period of time, perhaps three or four widely spaced measurements on each person will be sufficient to capture the shape and direction of the change. But, if the trajectory of individual change is more complex, then many more closely spaced measurements may be required. (p. 674)

In addition, to be true to a complexity approach, any longitudinal study must be set up to capture variability at various levels and timescales, from the general shape of the development process over a long period of time to the short-term variability that takes place between data collection intervals, to the within-session variability that inevitably arises. Van Geert and van Dijk (2002) argued that a study should attend to all timescales given that variability may be different on each scale: For example, "a developmental variable may be slowly oscillating while gradually growing, while another variable may increase discontinuously with sharp day-to-day fluctuations" (p. 346).

Capturing variability can take advantage of powerful computational tools now available. Van Geert and van Dijk (2002) showed how we can use computerized databases, graphing, and statistics to track complex patterns of variation in second language learners over time. Cameron and Stelma (2004) showed how cumulative frequency graphs and other types of visual data displays can assist analysis of the dynamics of discourse. Techniques, statistical and otherwise, used in analysis need to be suitable for genuinely longitudinal data rather than cross-sectional comparisons. We need to adopt and develop more appropriate ways of analysis to allow for the nonlinearity of the process, such as multivariate time-series modeling, growth curve analysis, latent factor modeling, and the like (Ellis, personal communication, 2006).

Methods of analysis of variability in dynamics systems, compiled by van Geert and van Dijk (2002), include "moving min-max" (p. 340) graphs, which, by plotting moving minima, maxima, and averages, show the data using the bandwidth of observed scores; graphs of score rangeschange in the range width might reflect various kinds of developmental phenomena; and standard deviations and coefficients of variation (see Verspoor, Lowie, \& van Dijk, this issue).

\section{Microdevelopment}

One approach to the study of change in behavior over a relatively short timescale is microdevelopment. It has become increasingly clear that in order to study "motors of change" (Thelen \& Corbetta, 2002, p. 59), we need not only longitudinal corpora, but also dense corpora that involve highly intensive sampling over short periods of time. Thelen and Corbetta suggested that the corpora that such an approach yields will not only allow us to fix the when of developmental milestones, but, what is important, the how of development by making development more transparent.

Thelen and Corbetta (2002) asserted that in traditional research, change is often inferred from an endpoint measurement. One assumption of researchers using a microdevelopment approach is that there are moments in the evolution of behavior where we can directly observe change happening. Furthermore, given that change works at multiple timescales, these small-scale changes can illuminate change at a longer timescale. A microdevelopment approach also allows us to plot multiple developmental routes to the same endpoint. This plotting allows us to capture important developmental differences among learners, both children and adults. From a dynamic systems perspective, variability needs to feature prominently in any account of development (Thelen \& Corbetta, 2002).

\section{Computer Modeling}

As articles in this issue demonstrate, computer simulations or models offer an important approach to researching complex dynamic systems. Although still in its infancy, modeling in applied linguistics shows great promise. This approach builds a computer model of the real-world 
complex system under investigation and takes it through multiple iterations, replicating change over time. The computer model is an analogy (or metaphor) of the real-world system, inevitably involving some reduction of complexity and some approximation. However, it is designed and adjusted so that the outcomes over time reflect what is known of the real-world system. Taking the model through successive iterations, which model evolution over time, or changing parameters and observing how outcomes change, which models changing conditions, allows the researcher to explore the workings of the model system and extrapolate back to the real-world system. In a further step, the processes of development seen in the model are examined and may be hypothesized as representing change in the actual system.

Turner (1997) contrasted the modeling process with conventional approaches to research:

Instead of creating a hypothesis, testing it on the experimental and observational facts until a counterexample shows its flaws, and then trying another, we can create an accurate facsimile of reality by successive tweakings of the variables and the connections among them, run the model in a computer as long as we like, check that its behavior continues to resemble that of the reality, and then read off what those parameters are. This procedure reverses the top-down theory-to-phenomena approach of classical science, and thus can provide an admirable complement to it. (pp. xxv-xxvi)

The activity of building a model is an important part of the research process because it requires explicit statements of theory and the most accurate empirical knowledge about the real systems and processes being modeled. Model building can thus be a very useful and formative activity. However, any model is only as good as the assumptions built into it. Inevitably, the model differs from the actual system, being idealized or simplified in some respects, approximated in others. This limitation raises new issues for applied linguistics around the validity and robustness of computer models of language development.

Articles in this issue demonstrate the potential of the two main types of models currently in use: neural network (or connectionist) models and agent-based models. Neural network models can replicate the learning of an individual brain and the emergence (or learning) of categories through self-organization. From very simple rules and initial conditions, the models can produce outcomes that closely resemble human learning and development in areas including vocabulary learning (Meara, 2006), past tense learning (Rumelhart \& McClelland, 1986), and the emergence of syntax from lexical learning (Elman, 1995). A major limitation of neural network models is their representation of the individual learner as isolated and as only cognitive, rather than as also an affective and social being.

Computer software such as "Swarm" (Minar, Burkhart, Langton, \& Askenazi, 1996) enables researchers to construct detailed, robust, agentbased models that involve a population of independent agents who interact in discrete events in a specific open-ended environment. Simulations consist of groups of interacting agents in a sequence of events that reflect the passage of time. Swarm simulations allow a researcher to investigate the global consequences of these local interactions and are beginning to advance our understanding of the evolution of language in social groups (Ke \& Holland, 2006), creolegenesis (Sattersfield, 2001), self-organizing vocabularies (Steels, 1996), and the acquisition of language from complex, situated input (Marocco, Cangelosi, \& Nolfi, 2003).

The validity of a simulation model is checked by comparing the outcomes produced by the model with the outcomes of the real-world human system. If the model reflects the real-world behavior, it is said to be valid. Gilbert and Troitzsch (2005) set out some of the issues around model validity. These issues include the uncertainty introduced by the stochastic nature of processes in both realworld and model systems; the path-dependence of simulations, that is, the sensitivity of a model to its initial conditions; the possibility that the simplification involved in model construction leads to its being incomplete in some important way; and the possibility that the real-world data used to build the model were themselves incorrect or based on incorrect assumptions. This last issue may be particularly important for our field because empirical data about language learning that we may need to build into the model may have been collected and analyzed under theoretical assumptions that differ greatly from those of the complex systems perspective. Cohen and Stewart (1994) made this point quite vividly in the context of biological models:

The hardest [errors] of all to spot are the implicit assumptions in the worldview that suggested the model. For example, suppose you're setting up a model of biological development based upon the idea of DNA as a message. You would naturally tend to focus upon such quantities as the amount of information in a creature's DNA string and the amount of information needed to describe the animal's physical form. If you then model development as a process of 
information transfer-lots of messages buzzing to and fro-you will implicitly have built a model in which information cannot be created. You will then be able to "prove" that humans can't develop a brain because the amount of information needed to list every connection in the human brain is a lot more than the total amount of information in human DNA.

But we do have brains .... Impeccable mathematics can produce nonsense if it is based on nonsensical assumptions .... Don't be impressed by mathematics just because you can't understand it. (p. 186)

Choices are made at each step in the building of a simulation model, from choosing and describing the real-world system to be modeled, through programming the rules for interaction among the agents in the model, to adjusting the parameters of the model and interpreting outcomes. Each choice can affect the model's validity and needs to be scrutinized and justified. Theoretical assumptions built into the model should be clearly stated in published papers so that readers can evaluate this aspect of validity.

The further step, of inferring back from the model to the real world about the processes of development, raises issues beyond what we might call outcome validity, as described previously. Similar outcomes may be produced by computer models and human systems through very different processes. For example, neural network models have an internal structure very different from that of a human learning system, and similarity of outcomes would not justify claiming similarity of internal processes. Process validity in simulation modeling is a more difficult construct than outcome validity. Inspecting the processes of simulation models may lead to areas worth investigating back in the real-world system, but claims of similarity should be treated with caution. We need to take care in how we label and talk about processes and parts of simulation models in order to avoid unwarranted or premature theoretical inferences: For example, van Geert's (this issue) choice of the term generators to describe processes established through model building prompts resonances with a specific theory of language development that might lead researchers' thinking in particular directions, whereas a more neutral term might be less constraining.

Simulation modeling of complex dynamic systems is clearly going to be very important for applied linguistics. Validity issues need careful attention and, because the methodology and technology are still unfamiliar and difficult for many researchers, the onus of validation will inevitably fall on the modelers themselves.

\section{Brain Imaging}

Technological advances in brain imaging, including improvements in the temporal and spatial resolution of electro-encephalographic and functional magnetic resonance images are allowing detailed descriptions of the dynamics of brain activity, promoting a shift of emphasis from knowledge as static representation stored in particular locations to knowledge as processing involving the dynamic mutual influence of interrelated types of information as they activate and inhibit each other over time (Ellis, personal communication, 2006). Brain imaging may contribute a useful tool to researching microdevelopment, although currently it is an expensive resource, often difficult for researchers to access.

\section{Combinations of Methodologies}

Combinations or blends of methodologies would seem to be particularly appropriate to the study of complex systems, allowing different levels and timescales to be investigated. We outline three possibilities.

Discourse Analysis and Corpus Linguistics. Large corpora of language use give us access to stabilized patterns and variability around them. Although we acknowledge that a corpus is a static collection of attested language and cannot show the dynamics of language as it unfolds in use or its future potential (Larsen-Freeman, 2006b), a corpus can serve to some extent as representative of the language resources of members of the speech community where it was collected. We can then combine corpus linguistics with close analysis of actual discourse, to trace the genesis and dynamics of language patterns, such as the conventionalization and signaling of metaphors (Cameron \& Deignan, 2003, 2006).

Second Language Acquisition and Corpus Linguistics. The field of second language acquisition (SLA) needs to make more use of computersearchable longitudinal corpora for addressing theoretical issues. Rutherford and Thomas (2001) and Myles (2005) advocated the use of Child Language Data Exchange System (CHILDES) tools for SLA research. Mellow (2006) illustrated the impact of large new computerized corpora such as CHILDES and TalkBank (MacWhinney, Bird, Cieri, \& Martell, 2004) on theories of second language learning. A corpus of adult English as a second language (ESL) learners in a classroom 
setting is also a powerful aid in helping us to understand adult language learning better (Reder, Harris, \& Setzler, 2003).

Second Language Acquisition and Conversation Analysis. Conversation analysis (CA) attends to the dynamics of talk on the microlevel timescale of seconds and minutes. In a recent special issue of The Modern Language Journal (Markee \& Kasper, 2004), it was argued that joining a CA perspective on interaction with a long-term view of language development holds great promise (LarsenFreeman, 2004; Kelly Hall, 2004). CA offers a rich description of "the most basic site of organized activity where learning can take place" (Mondada \& Pekarek Doehler, 2004, p. 502). If these analyses were to be done with a sufficient density so that retrospective microdevelopmental analyses could be conducted, it would offer another means of connecting synchronic dynamism to its over-time counterpart.

\section{CONCLUSION}

Clearly, each of the approaches just discussed has advantages and drawbacks. CA offers an indepth view of conversational interaction, but it ignores any insights that a conscious introspection would permit. Corpus linguistics offers rich usage data, but the data are attested; they do not demonstrate the potential of the system. Other approaches, such as brain imaging and neural network modeling, illustrate or simulate the dynamic patterns of brain activity, but do so by isolating the learner's brain from society and from its normal ecology of function. Computer models might be more encompassing in this regard, in allowing for a social interactive dimension, but they, too, involve ecologically reduced ways of representing reality.

We began this article by asking whether the methods of inquiry of the natural sciences would work for applied linguists whose theoretical commitment is to understanding complex, dynamic systems. We have pointed out that different assumptions (e.g., about causality) underlie traditional research methods used in both the natural and the social sciences and have discussed those methods that are perhaps the most suitable for a complexity theory perspective.

What we can aspire to at this point is to entertain the principles we have enumerated earlier in this article while blending and adapting methods, a trend that is increasingly adopted across the social sciences. It should not be surprising that researchers are entertaining the possibility of using multiple blended methods. It is, after all, a pragmatic solution to the demands of a theoretical perspective that seeks to understand the dynamics of change in complex systems.

\section{NOTES}

${ }^{1}$ We are grateful for discussions we have had on these issues with colleagues, especially Nick Ellis and Ke JinYun. Also, portions of this article appear as a chapter in Larsen-Freeman and Cameron's (2008) book.

${ }^{2}$ It should be noted that self-organization is not some kind of automatic self-improvement, but is neutral as to whether its outcome is better or worse than the previous state.

${ }^{3}$ Gaddis is an historian, not an applied linguist, but he is insightful about the nature of research in a way that we think is helpful to applied linguists. We thank Gad Lim for bringing this book to our attention.

${ }^{4} \mathrm{An}$ affordance is an opportunity for use or interaction that some object or state of affairs presents to a certain kind of agent. "For example, to a human a chair affords sitting, but to a woodpecker it may afford something quite different" (Clark, 1997, p. 172).

\section{REFERENCES}

Agar, M. (2004). We have met the other and we're all nonlinear: Ethnography as a nonlinear dynamic system. Complexity, 10(2), 16-24.

Atkinson, D. (2002). Toward a sociocognitive approach to second language acquisition. Modern Language Journal, 86, 525-545.

Bak, P. (1997). How nature works: The science of selforganized criticality. New York: Oxford University Press.

Barab, S. (2006). Design-based research: A methodological toolkit for the learning scientist. In R. Sawyer (Ed.), The Cambridge handbook of the learning sciences (pp. 153-169). Cambridge: Cambridge University Press.

Baskerville, R., \& Wood-Harper, T. (1996). A critical perspective on action research as a method for information systems research. Journal of Information Technology, 11, 235-246.

Byrne, D. (2002). Interpreting quantitative data. London: Sage.

Cameron, L. (2003). Metaphor in educational discourse. London: Continuum.

Cameron, L., \& Deignan, A. (2003). Using large and small corpora to investigate tuning devices around metaphor in spoken discourse. Metaphor and Symbol, 18, 149-160.

Cameron, L., \& Deignan, A. (2006). The emergence of metaphor in discourse. Applied Linguistics, 27, 671-690. 
Cameron, L., \& Stelma, J. (2004). Metaphor clusters in discourse. Journal of Applied Linguistics, 1, 7-36.

Clark, A. (1997). Being there. Cambridge, MA: MIT Press.

Cohen, J., \& Stewart, I. (1994). The collapse of chaos. London: Viking.

Davis, K., \& Lazaraton, A. (Eds.). (1995). Qualitative research in ESOL (Special Issue). TESOL Quarterly, 29(3).

de Bot, K., Lowie, W., \& Verspoor, M. (2007). A dynamic systems approach to second language acquisition. Bilingualism: Language and Cognition, 10, 7-21, 51-55.

Ellis, N., \& Larsen-Freeman, D. (2006). Language emergence: Implications for applied linguistics. Applied Linguistics, 27, 558-589.

Elman, J. (1995). Language as a dynamical system. In R. Port \& T. van Gelder (Eds.), Mind as motion: Explorations in the dynamics of cognition (pp. 195223). Cambridge, MA: MIT Press.

Fischer, K. W., \& Bidell, T. (1998). Dynamic development of psychological structures in action and thought. In R. M. Lerner \& W. Damon (Eds.), Handbook of child psychology: Vol. 1. Theoretical models of human development (pp. 467-651). New York: Wiley.

Gaddis, J. L. (2002). The landscape of history. Oxford: Oxford University Press.

Gilbert, N., \& Troitzsch, K. (2005). Simulation for the social scientist (2nd ed.). Maidenhead, UK: Open University Press.

Goffman, E. (1974). Frame analysis. London: Harper and Row.

Holliday, A. (1996). Developing a sociological imagination: Expanding ethnography in international English language education. Applied Linguistics, 17, 234-255.

Jacob, E. (1992). Culture, context, and cognition. In M. D. Lecompte, W. L. Millroy, \& J. Preissle (Eds.), The handbook of qualitative research in education (pp. 292-335). San Diego, CA: Academic Press.

Ke, J.-Y., \& Holland, J. (2006). Language origin from an emergentist perspective. Applied Linguistics, 27, 691-716.

Kelly Hall, J. (2004). Language learning as an interactional achievement. Modern Language Journal, 88, 607-612.

Kemmis, S. (2001). Exploring the relevance of critical theory for action research: Emancipatory action research in the footsteps of Jurgen Habermas. In P. Reason \& H. Bradbury (Eds.), Handbook of action research: Participative inquiry and practice (pp. 91102). London: Sage.

Kramsch, C. (Ed.). 2002. Language acquisition and language socialization. Ecological perspectives. London: Continuum.

Lantolf, J. (Ed.). (1994). Sociocultural theory and second language learning (Special issue). Modern Language Journal, 78(4).

Larsen-Freeman, D. (1997). Chaos/complexity science and second language acquisition. Applied Linguistics, 26, 141-165.
Larsen-Freeman, D. (2004). CA for SLA? It all depends. Modern Language Journal, 88, 603-607.

Larsen-Freeman, D. (2006a). The emergence of complexity, fluency, and accuracy in the oral and written production of five Chinese learners of English. Applied Linguistics, 27, 590-619.

Larsen-Freeman, D. (2006b). Functional grammar: On the value and limitations of dependability, inference, and generalizability. In M. ChalhoubDeville, C. Chapelle, \& P. Duff (Eds.), Inference and generalizability in applied linguistics (pp. 115-133). Amsterdam/Philadelphia: John Benjamins.

Larsen-Freeman, D., \& Cameron, L. (2008). Complex systems and applied linguistics. Oxford: Oxford University Press.

Leather, J., \& van Dam, J. (Eds.). (2003). Ecology of language acquisition. Dordrecht, The Netherlands: Kluwer Academic Publishers.

Lemke, J. (2002). Language development and identity: Multiple timescales in the social ecology of learning. In C. Kramsch (Ed.), Language acquisition and language socialization (pp. 68-87). London: Continuum.

Lobato, J. (2003). How design experiments can inform a rethinking of transfer and vice versa. Educational Researcher, 32, 17-20.

MacWhinney, B., Bird, S., Cieri, C., \& Martell, C. (2004). TalkBank: Building on open unified multimodal database of communicative interaction. In Proceedings of the Languages Resources and Evaluation Conference, 2004 (pp. 525-528). Lisbon, Portugal: Language Resources and Evaluation Conference.

Markee, N., \& Kasper, G. (Eds.). (2004). Classroom talks [Special issue]. Modern Language Journal, 88(4).

Marocco, D., Cangelosi, A., \& Nolfi, S. (2003). The emergence of communication in evolutionary robots. Philosophical Transactions of the Royal Society of London A, 361, 2397-2421.

Meara, P. (2006). Emergent properties of multilingual lexicons. Applied Linguistics, 27, 620-644.

Mellow, J. D. (2006). The emergence of second language syntax: A case study of the acquisition of relative clauses. Applied Linguistics, 27, 645-670.

Minar, N., Burkhart, R., Langton, C., \& Askenazi, M. (1996). The swarm simulation system: A toolkit for building multi-agent simulations. (Working Paper No. 96-06-042). Santa Fe, NM: Santa Fe Institute.

Mondada, L., \& Pekarek Doehler, S. (2004). Second language acquisition as situated practice: Task accomplishment in the French second language classroom. Modern Language Journal, 88, 501-518.

Myles, F. (2005). Interlanguage corpora and second language acquisition research. Second Language Research, 21, 373-391.

Newman, D. (1990). Opportunities for research on the organizational impact of school computers. Educational Researcher, 19, 8-13.

Ortega, L., \& Iberri-Shea, G. (2005). Longitudinal research in second language acquisition: Recent trends and future directions. Annual Review of Applied Linguistics, 25, 26-46. 
Ramanathan, V., \& Atkinson, D. (1999). Ethnographic approaches and methods in L2 writing research: A critical guide and review. Applied Linguistics, 20, 44-70.

Reder, S., Harris, K., \& Setzler, K. (2003). A multimedia adult learner corpus. TESOL Quarterly, 37, 546557.

Reinking, D., \& Watkins, J. (2000). A formative experiment investigating the use of multimedia book reviews to increase elementary students' independent reading. The Reading Research Quarterly, 35, 384-419.

Rumelhart, D., \& McClelland, J. (1986). On learning the past tenses of English verbs. In J. McClelland, D. Rumelhart, \& the PDP Research Group (Eds.), Parallel distributed processing: Explorations in the microstructure of cognition: Vol. 2. Psychological and biological models (pp. 216-271). Cambridge, MA: MIT Press.

Rutherford, W., \& Thomas, M. (2001). The child language data exchange system. Second Language Research, 17, 195-212.

Salthe, S. (1993). Development and evolution. Cambridge: MIT Press.

Sattersfield, T. (2001). Toward a sociogenetic solution: Examining language formation processes through SWARM modeling. Social Science Computer Review, 19, 281-295.

Sealey, A., \& Carter, B. (2004). Applied linguistics as social science. London: Continuum.

Steels, L. (1996). A self-organizing spatial vocabulary. Artificial Life Journal, 2/3, 319-332.

Thelen, E., \& Corbetta, D. (2002). Microdevelopment and dynamic systems: Applications to infant motor development. In N. Granott \& J. Parziale (Eds.), Microdevelopment: Transition processes in development and learning (pp. 59-79). Cambridge: Cambridge University Press.

Thelen, E., \& Smith, L. (1994). A dynamic systems approach to the development of cognition and action. Cambridge, MA: MIT Press.

Turner, F. (1997). Foreward. In R. Eve, S. Horsfall, \& M. Lee (Eds.), Chaos, complexity, and sociology: Myths, models, and theories (pp. xi-xxviii). Thousand Oaks, CA: Sage.

van Geert. (2008). The dynamic systems approach in the study of L1 and L2 acquisition: An introduction. Modern Language Journal, 92, 179-199.

van Geert, P., \& Steenbeek, H. (in press). A complexity and dynamic systems approach to development assessment, modeling and research. In K. W. Fischer, A. Battro, \& P. Lena (Eds.), The educated brain. Cambridge: Cambridge University Press.

van Geert, P., \& van Dijk, M. (2002). Focus on variability: New tools to study intra-individual variability in developmental data. Infant Behavior E Development, 25, 340-374.

van Lier, L. (2004). The ecology and semiotics of language learning. Dordrecht, the Netherlands: Kluwer Academic.

Verspoor, M., Lowie, W., \& van Dijk, M. (2008). Variability in second language development from a Dynamic Systems perspective. Modern Language Journal, 92, 214-231.

Willett, J. B. (1994). Measuring change more effectively by modeling individual growth over time. International encyclopedia of education (2nd ed., pp. 671678). Amsterdam: Elsevier.

\section{New $M L J$ Web Site}

The Modern Language Journal Web site has moved to its new location at http://mlj.miis.edu/. The old site, located at http://polyglot.lss.wisc.edu/mlj, is no longer active.

The new Web site is nearly up to date, but if you notice anything that needs to be fixed or updated, or if you have any suggestions for improvement, please contact the editorial staff at mlj@miis.edu with your comments. 